\title{
A low background ionisation chamber for alpha-spectroscopy
}

\author{
Heinrich Wilsenach ${ }^{1, a}$, Kai Zuber ${ }^{1}$, Felix Krüger ${ }^{1}$, Andreas Hartmann² ${ }^{2}$, and Manfred Sobiella ${ }^{2}$ \\ ${ }^{1}$ Institut für Kern- und Teilchenphysik, TU-Dresden, Dresden, Germany \\ 2 Helmholtz Zentrum Dresden-Rossendorf (HZDR), Dresden, Germany
}

\begin{abstract}
The goal of designing a low background ionisation chamber is to measure long lived $\alpha$-decay halflives which might interfere with rare event searches. Such decays play a part in many fields in nuclear physics and are difficult to measure. A lot of Geiger-Nutall studies also depend on them. Among others the research is specifically aimed at the precision measurements of $\alpha$ emitters mainly within the Lanthanide region. The excellent energy resolution would also allow to search for excited states in $\alpha$-decays. To achieve this goal a gridded ionisation chamber was constructed using the Frisch-Grid design. A background rate of only 10.9(6) counts per day has been achieved in the energy region of $1 \mathrm{MeV}$ to $9 \mathrm{MeV}$ and improvements are possible. This low background rate and size of the chamber allows precision measurements of long living alpha decays with half-lives in the region of $1 \times 10^{15}$ years.
\end{abstract}

\section{Introduction}

One of the most important tools used to understand the mechanism of the nucleus has always been $\alpha$-decays. The combination of the rate and energy of the $\alpha$-decay yields information on the inner structure of the nucleus.

In an $\alpha$-decay a ${ }^{4} \mathrm{He}$ particle is preformed inside the atomic core. The $\alpha$ particle is then trapped in the potential of the nucleus. There is a small probability of the $\alpha$ particle tunnelling out through the potential barrier. There is no limit on how many times the $\alpha$ particle can interact with the potential barrier. This fact allows the decay to occur, even though the probability of tunnelling is small.

This mechanism of the decay gives a strong relationship between the Q-value of the decay and its halflife. This relationship was first observed by $\mathrm{H}$. Geiger and J.M. Nuttall [1] in 1911. They realised that a relationship between the $\alpha$-decay's energy and its half-life existed, though they were limited by then number of $\alpha$-decaying isotopes discovered at the time. A more complete relation was determined by V.E. Viola and G.T. Seaborg [2] in 1966. Today the most recent update is given in [3], with a microscopic determination of the Viola-Seaborg parameters.

There is no consensus of the laws determined above and the half-lives of low energy $\alpha$-decays. One of the main reasons of this is the quality of data for long lived $\alpha$-decaying isotopes. More precise measurements of the half-lives of long lives $\alpha$-decays would help towards the determination of a universal decay law.

Another area of interest this the remeasurement of isotopes used in geo and cosmochronometry. Three isotopes of interest are ${ }^{147} \mathrm{Sm},{ }^{146} \mathrm{Sm}$ and ${ }^{190} \mathrm{Pt}$. There is some tension to be resolved with the current half-life values for the above mentioned isotopes. This situation would be helped with the measurement of the decay rate of thin well characterised samples. The rate is then combined

a e-mail: heinrich.wilsenach@mailbox.tu-dresden.de with the number of isotopes in the samples to determine the half-life of the isotope.

\section{Detector}

The detector design chosen is that of the Twin FrischGrid [4] ionisation chamber (TF-GIC). The TF-GIC has the advantage of a high efficiency as well as excellent noise rejection and energy resolution. The schematic drawing of the design of the chamber can be seen in Fig. 1. The chamber is built out of radio pure materials for low intrinsic background. The design of the TF-GIC allows for fast pulses $(\sim \mu \mathrm{s})$ to be recorded with a CAEN:N6724 digitiser after being amplified with a CAEN:A1422 preAmplifier. The pulse shapes are stored in ROOT files for further analysis.

The TF-GIC uses the ionisation of P10, this is a gas composed of $90 \%$ argon and $10 \%$ methane. The advantage of this gas is that it only requires a voltage of around 1,200 volts to have a suitable electron drift velocity $(\sim 5 \mathrm{~cm} / \mu \mathrm{s})$. The gas is also commonly used in industry and is relatively inexpensive as well as very pure.

The main chamber is divided into two parts, each functioning as a mirrored detector sharing an anode. This is used to reject noise pulses as well as veto any events caused by cosmic-rays. Each chamber is then divided into two regions by gridded wires. The interaction region is defined as the space between the cathode and the grid. This is the region where the $\alpha$ particle deposits all of its energy due to ionisation inside of the gas. The second region is the detection region and is the space between the grid and anode. The grids are specially designed to shield the anode from any signal induced from charges moving in the interaction region. The main signal induced on the anode only then depends on the movement on charges between the grid and the anode. This gives the signal a fast decay time, and also means that the amplitude of the anode pulse is independent of the angle of the decay. Moreover, the full 


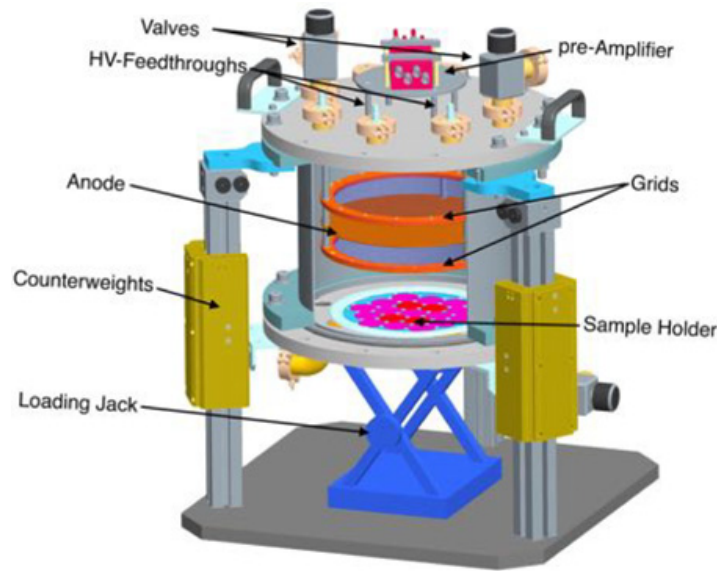

Figure 1. Schematic drawing of the TF-GIC. The schematic shows the position of the grids and the anode as well as the loading system.

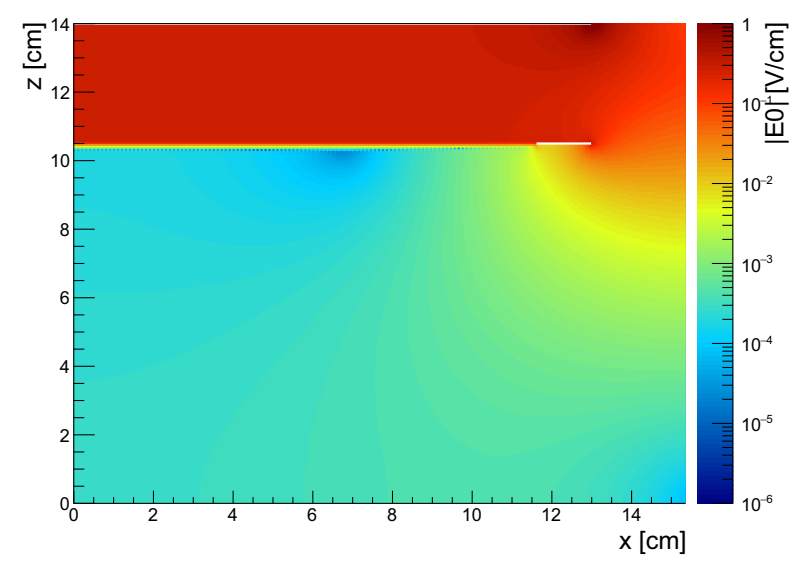

Figure 2. A graph showing the weighed field of the anode. The red region here is the detection region and the blue region is the interaction region. The grid is the horizontal line located at $\mathrm{z}=10.5 \mathrm{~cm}$. It is clear to see that the grid is shielding the anode from the electrons moving through the detection region.

signal collection time only depends on the electrons, which move much faster through the gas.

The signal induced on an electrode can be understood through the application of the Shockley-Ramo theorem $[5,6]$. The theorem states that the charge induced on an electrode by a moving charge is given by the following equation,

$$
I(t)=q \cdot \bar{v} \cdot \bar{E}_{0},
$$

where $q$ is the charge of the signal carrier, $\bar{v}$ is the velocity in the direction of motion and $\bar{E}_{0}$ is the weighted field of the electrode of interest. The weighed field is produced by solving the Laplace equation for the system, setting the electrode of interest to one and all other electrodes to zero. An example of such a weighed field can be seen in Fig. 2. Here the shielding effect of the grid is easy to see.

It is shown in [7] that the centre of charge of an event can be obtained from the pulse shape. This is given by the ratio of the grid and anode pulse. This is shown in Fig. 3. Figure 4 shows the position as a function of energy for an ${ }^{241} \mathrm{Am}$ sample. The solid line here is the maximum position that an $\alpha$ particle can have with a certain energy. This is derived using SRIM [8] and the parameters for P10

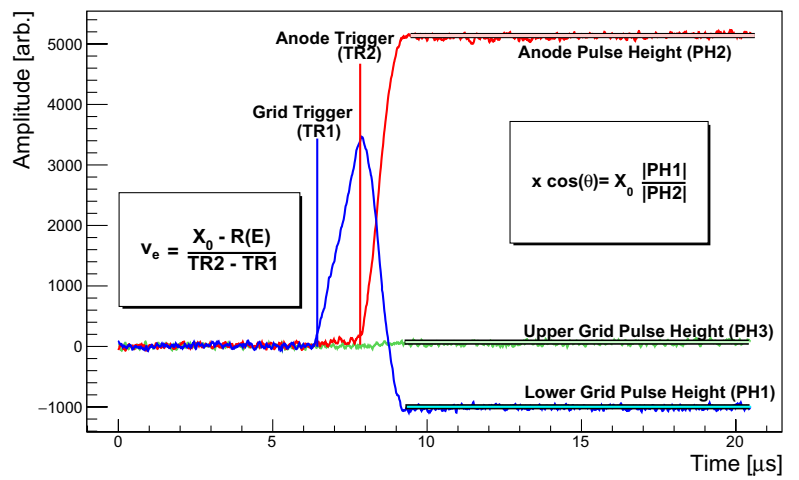

Figure 3. Graph showing a typical pulse shape of an event in the chamber. All of the cut parameters are marked on the pulse.

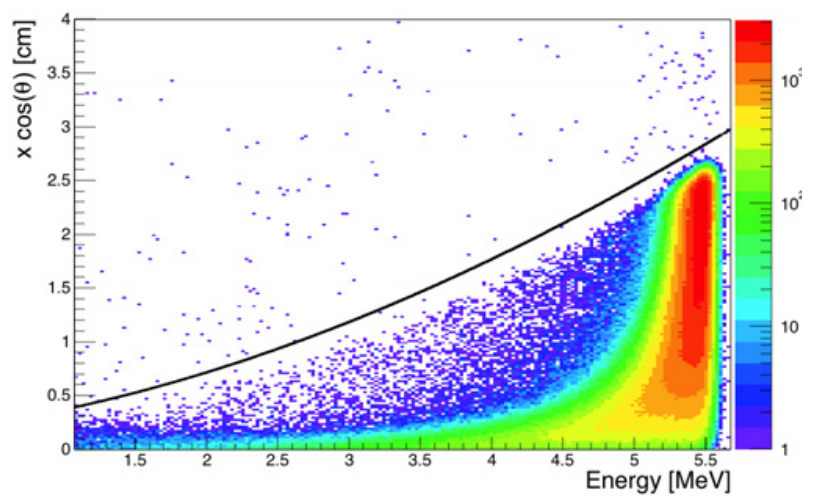

Figure 4. The vertical position of the centre of charge for each pulse. The solid line is the maximum of the cut.

gas. The position information is combined with the trigger information to find the velocity of the signal carriers in the gas. These values are then used to determine if an event is signal coming from the bottom of the chamber.

An important parameter of the chamber is the total detection efficiency. This efficiency is used to determine the true rate of signal in the chamber. Without the true rate of signal in the chamber it is not possible to accurately measure the half-life of an isotope. Background pulse shapes come from two different sources. The first can be caused by alpha events that occur on the grids, anode and walls of the chamber. These are cut as they are unrelated to signal events. The second is from electronic noise that can be cause by microphonics or disturbances from the high voltage supplies.

The total efficiency of the chamber in detecting signal events are a combination of two efficiencies. The first due to pulse shape discrimination. This efficiency was found by sorting pulses by eye into a library of 6066 signal pulses, 419 noise pulses and 1383 pulses from background events. The sorted pulses are then passed through the cuts to determine how many signal pulses have been identified correctly. Moreover, the cut efficiency is weakened if a noise pulse or pulses from background give false positives. This was determined to be $99.3 \%$.

This is then combined with the detection efficiency to find the total detection efficiency. The detection efficiency of the chamber was measured with a calibrated ${ }^{241} \mathrm{Am}$ source. The rate of this source was high enough that the rate of false positives due to noise and background events could be neglected. The pulses are again checked by eye to 


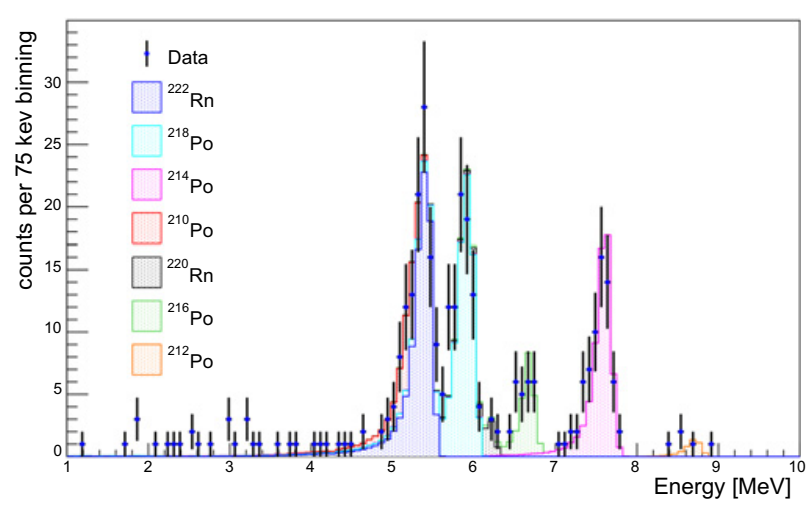

Figure 5. A histogram of the background from the thorium and uranium decay chains. The run laster 30.8 days.

make sure that there are no false positives. The rate in the chamber is then compared with the calibrated activity of the source to find the detection efficiency. The combination of the detection efficiency and the cut efficiency is then combined into the total detection efficiency, this was found to be $98.6(22) \%$. The error here is dominated by the uncertainty on the activity of the calibrated source and is the main systematic uncertainty on every measurement.

The energy and resolution of the chamber was calibrated with an ${ }^{239} \mathrm{Pu},{ }^{241} \mathrm{Am},{ }^{244} \mathrm{Cm}$ mixed $\alpha$ standard for high energy and a ${ }^{147} \mathrm{Sm}$ sample for lower energy. More detail about the design and performance can be found in [9].

\section{Measurements}

Even though the TF-GIC was built out of radio pure materials and the cuts are designed to have a high background discrimination efficiency, some background events are still present. Figure 5 shows the fitted energy spectrum of a continuous 30.8 day run. The chamber was not opened in this period. The main part of this background comes from the decay of ${ }^{222} \mathrm{Rn}$ into its daughter nuclei. This isotope is present in ambient air and gets into the chamber when the samples are loaded. Figure 6 shows the time profile of the background. It is clear that the contamination coming from ${ }^{222} \mathrm{Rn}$ does not die away. This is due to the chamber not being completely vacuum tight. When the chamber expands and cools through the day night period, a small amount of ${ }^{222} \mathrm{Rn}$ enters the chamber. The decay of ${ }^{222} \mathrm{Rn}$ to ${ }^{218} \mathrm{Po}$ is detected using a time and energy coincidence cut, this is displayed as a the black lines in Fig. 6. There are also lines visible from the decay of ${ }^{220} \mathrm{Rn}$ from the ${ }^{232} \mathrm{Th}$ decay chain. This is probably due to some contaminated dust that entered the chamber through the loading process. The decay chain of ${ }^{224} \mathrm{Ra}$ to ${ }^{212} \mathrm{~Pb}$ through ${ }^{220} \mathrm{Rn}$ and ${ }^{216} \mathrm{Po}$ was also searched for by using a time and energy coincidence cut. Unfortunately too few events were distinguished to determine the half life with any accuracy. The total count rate from 9 to $1 \mathrm{MeV}$ is measured to be 10.9(6) c.p.d.

The remeasurement of the decay of ${ }^{147} \mathrm{Sm}$ is one of the goals of this project. This is because ${ }^{147} \mathrm{Sm}$ has long halflife as well as a relatively high natural abundance. This means that samples can be made at low cost and with good quality. Four samples were produced using pulsed laser deposition in ultra high vacuum. To determine the effect of

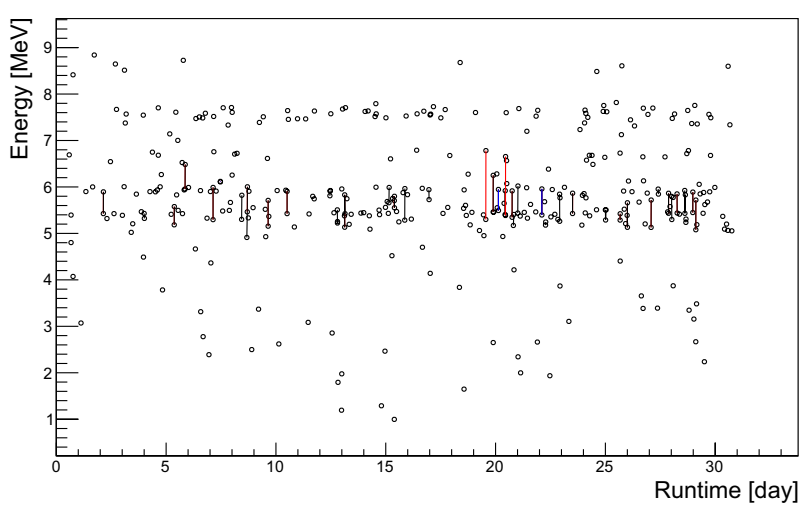

Figure 6. A graph showing the energy as a function of time of the the background run. A coincidence cut has been used to identify specific decays. The black lines are ${ }^{222} \mathrm{Rn}$, the red lines are ${ }^{224} \mathrm{Ra}$ and the blue lines are ${ }^{220} \mathrm{Rn}$.

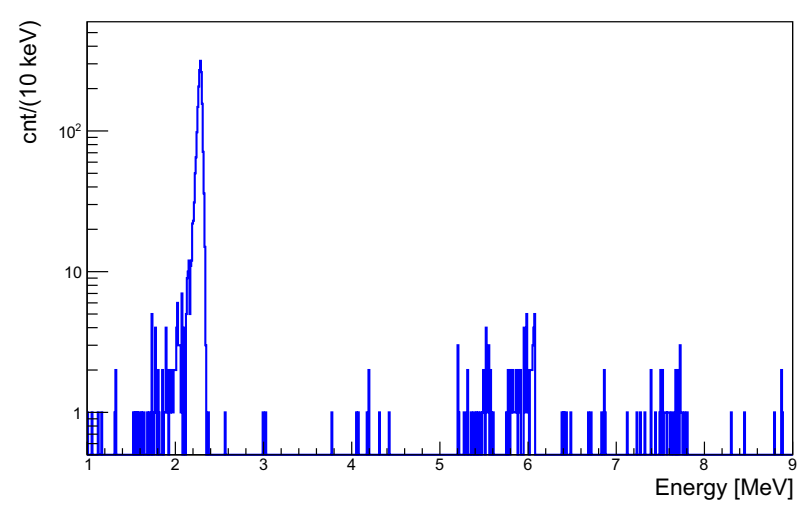

Figure 7. An energy spectrum of ${ }^{147} \mathrm{Sm}$ sample. The background can be seen above the peak, and is shown to be relatively small compared with the signal. The run lasted for 19 days.

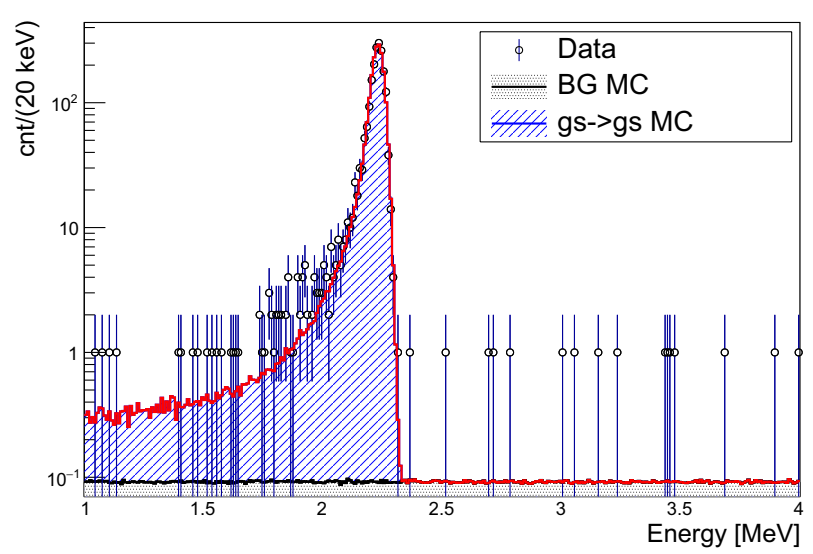

Figure 8. An energy spectrum of ${ }^{147} \mathrm{Sm}$ with a $41(1) \mathrm{nm}$ thick sample.

the tailing caused by self absorption, a range of thicknesses were chosen. Their thicknesses were 35.2(7) nm, 41(1) nm, 226(2) nm and 904(7) nm. Figure 7 shows the energy spectrum of the thinnest sample relative to the background on a logarithmic plot.

The energy spectra are fitted with a Monte Carlo spectrum. The simulation was made using the GEANT4 package [10]. It is clear from the spectra that the simulation describes the data very well, and is also able to describe the whole range of thicknesses. The fitting procedure is also 


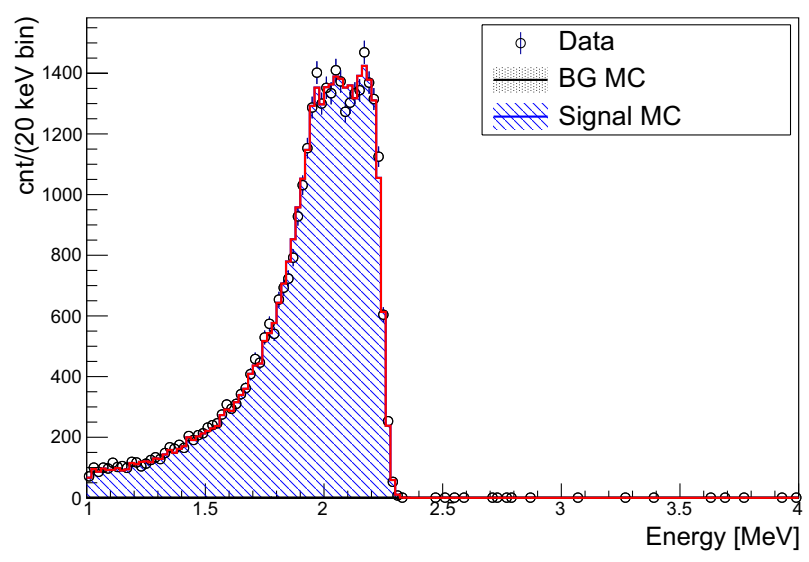

Figure 9. The comparison energy spectrum of a 904(7) nm thick sample. Here the effect of self absorption can be seen very clearly. The solid red line is the total fit from simulation. A flat background is assumed here.

able to determine the thickness from the tailing, this is in agreement with the measured values.

\section{Conclusions}

This work has discussed the installation and operation of an $\alpha$ chamber for long lived isotopes. The development of pulse shape analysis to suppress background has also been shown. The chamber was also calibrated, and the efficiency determined. The chamber was shown to have a very low internal background contamination which makes it ideal for measuring long-lived $\alpha$ decaying isotopes as even a low signal rate can be accurately measured. Some preliminary measurements of ${ }^{147} \mathrm{Sm}$ are also presented here.

The next stage of this project is to combine the values from all of the measurements of ${ }^{147} \mathrm{Sm}$ into a half-life. This will give an indication of the performance of the chamber and will show the accuracy to which it can measure, though it is already known that the greatest limit in accuracy is the knowledge of the detector efficiency. The systematic error depends on the uncertainty of the calibration source. This gives a $2.2 \%$ systematic uncertainty on the chambers total detection efficiency.

The construction of this project was funded by the BMBF grant 02NUK13A HZDR and 02NUK13B TUD. The author would like to thank the Humboldt-Universität zu Berlin "Masse Spektrum Symmetrie" Graduiertenkolleg for funding the costs of the conference and travel.

\section{References}

[1] H. Geiger, J. Nuttall, Philosophical Magazine Series 22, 613 (1911)

[2] V. Viola, G. Seaborg, Journal of Inorganic and Nuclear Chemistry 28, 741 (1966)

[3] B. Sahu, S. Bhoi, Phys. Rev. C 93, 044301 (2016)

[4] O. Frisch, British Atomic Energy Report No. BR-49 (1944)

[5] W. Shockley, Journal of Applied Physics 9, 635 (1938)

[6] S. Ramo, Proceedings of the IRE 27, 584 (1939)

[7] A. Al-Adili, F.J. Hambsch, R. Bencardino, S. Pomp, S. Oberstedt, S. Zeynalov, Nuclear Instruments and Methods in Physics Research Section A: Accelerators, Spectrometers, Detectors and Associated Equipment 671, 103 (2012)

[8] J.F. Ziegler, M. Ziegler, J. Biersack, Nuclear Instruments and Methods in Physics Research Section B: Beam Interactions with Materials and Atoms 268, 1818 (2010), 19th International Conference on Ion Beam Analysis

[9] A. Hartmann, J. Hutsch, F. Krüger, M. Sobiella, H. Wilsenach, K. Zuber, NIM A 814, 12 (2016)

[10] S. Agostinelli et al. (GEANT4), Nucl. Instrum. Meth. A506, 250 (2003) 